\title{
Modal properties of a strip-loaded horizontal slot waveguide
}

\author{
Ségolène Pélisset ${ }^{*} \mathbb{D}$, Janne Laukkanen, Markku Kuittinen, Seppo Honkanen and Matthieu Roussey
}

\begin{abstract}
Background: Channel waveguides have been developed for years to comply with the need for smaller footprint and better integration in photonics circuits. However, they are still suffering from fabrication complexity and optical losses. These two limitations can be addressed by combining two types of channel waveguides, namely strip-loaded and slot waveguide.

Methods: We present a systematic study of the geometrical parameters of a strip-loaded horizontal slot waveguide and their influence on the characteristics of the device. Simulations and experiments are carried out to understand the behavior of the electromagnetic field inside such a waveguide. In particular, the influence of the high and low refractive index layers and the loading-strip on the key characteristics of the structure, i.e., the confinement factor and the effective index, are investigated theoretically and compared with experimental values.

Results: The main properties of this waveguide platform are a low lateral index contrast, a high vertical field confinement, and low-propagation losses $(1.4 \mathrm{~dB} / \mathrm{cm})$.

Conclusions: Our results show that this platform is highly versatile, easy to fabricate and low-losses. We also show that the geometry of the mode can be tuned to suit the application.
\end{abstract}

Keywords: Optical waveguides, Thin film devices, Nanophotonics, Propagation losses

\section{Background}

Since it was introduced in 2004 by Almeida et al. [1], the slot waveguide has arisen a huge interest for photonic integrated circuits from telecommunication applications, to highly non-linear devices, and to sensing [2-6]. The potential of this structure lays in the high light confinement achievable in a tiny gap of low refractive index. This low refractive index expands the choice of materials to those usually not considered for guiding purposes. However, one of the main drawbacks of this structure is that the field is confined at the boundaries between high and low index materials, which implies a high sensitivity to the surface quality, especially the sidewall roughness of the rails [7]. The scattering that occurs due to roughness leads to high propagation losses $[8,9]$.

The concept of a slot waveguide lies in the discontinuity of the normal component of the electric field at the boundaries of two high index narrow waveguides, which yields a highly-confined field in the low index gap region

\footnotetext{
* Correspondence: segolene.pelisset@uef.fi

Institute of Photonics, University of Eastern Finland, 80101 Joensuu, Finland
}

(slot) when these two rails are close enough to each other. Losses due to the surfaces arise mostly because of the need, in integrated optics, of channel waveguides. The slot can be vertical [10] or horizontal [11, 12], and an etching step is required, which enables the two-dimensional confinement of light, and therefore creating the channel. This last step is often the source for losses.

Such an inconvenience can be easily avoided by using another concept: the strip-loaded waveguide [13]. A strip pattern on top of a slab waveguide will increase the effective index of the propagating mode leading to the creation of the required channel. We have applied this concept and demonstrated the principle by the creation of a strip-loaded horizontal slot waveguide in Ref. [14]. Atomic layer deposition (ALD) was used to fabricate the film-stack forming the slot and electron beam lithography (EBL) to pattern the strip. EBL is an expensive technique and difficult to adapt to industrial processing needs. However, we have shown that the patterning can be easily done by nano-imprint lithography (NIL) due to the relaxed fabrication constraints of the strip. 
In addition to its low propagation losses and high integrability, the strip-loaded slot waveguide (SLSW) offers numerous advantages in terms of degree of freedom compared to other waveguide types. The effective index, the symmetry, and the confinement of the guided mode can be controlled by geometrically tuning either the layers composing the slot or the shape and size of the loading-strip. In this paper, we present a thorough investigation of such a waveguide by studying the effect of different parameters by simulations and experiments.

The SLSW structure is sketched in Fig. 1a. It is a horizontal slot waveguide with a polymer loading-strip patterned on top to create the required effective index difference for the establishment of a channel waveguide. Light is confined both by the slot waveguide and by the loadingstrip. It yields a higher vertical confinement, due to the slot, than in the lateral direction. Examples of mode distributions are given in Fig. $1 \mathrm{~b}$ for an optimized single-mode waveguide $\left(\mathrm{TM}_{00}\right)$, for a waveguide with a wide loadingstrip leading to multiple lateral modes ( $\mathrm{TM}_{10}$ is shown), and for a waveguide with a thick loading-strip supporting multiple vertical modes ( $\mathrm{TM}_{01}$ is shown).

As mentioned, the slot waveguide is composed of two high refractive index material (HI) layers separated by a thin film of a lower index material (LI), called the slot region. This provides the vertical confinement in our case. The confinement within the slot is coming from merging of two single-rail modes together (totally decoupled initially). Each single-rail sustains a mode having evanescent tails extending on both sides. When the two rails are close enough the constructive superimposition of their evanescent tails become predominant over the rail mode. A good estimation of this critical distance can be determined by observing the characteristic length $1 / \gamma_{s}$ of the resulting mode profile inside the slot $[1,15]$. The slot thickness has to be smaller than the characteristic length, with $\gamma_{s}=\sqrt{\beta^{2}-k_{0}^{2} n_{s}^{2}}$ and $\beta$ the propagation constant of the slot mode. The thickness of the two
HI-layers has to be optimized for a specific wavelength in order to prevent light being too much confined in the high index regions. Also note that the propagation constant $\beta$ depends on the thickness of the HI-layers, and careful simulations have to be performed, taking into account the dependence of the characteristics of the mode with all the geometrical parameters at the same time.

The lateral light confinement is achieved by adding a strip on top of the topmost high index layer (Fig. 1c, profile in $x$-direction). The strip locally increases the effective index of the modes propagating in the horizontal slot waveguide below it, therefore creating the channel waveguide. The thickness and the width of the loadingstrip are crucial parameters. In order to keep light well confined within the slot, it is necessary to maintain the strip thin enough, but on the other hand, a too thin strip will not induce an appreciable change of effective index of the slot mode. The strip width acts directly on the amount of modes sustained by the waveguide and on their shape, see Fig. $1 b$.

We have already demonstrated the versatility of the SLSW platform for various types of photonic applications [14]. The present article aims at describing the structure in details and showing the effect of all the geometrical parameters on the different characteristics of the guided mode. The role of the platform is to concentrate as much as possible the field inside the silicon dioxide slot below the strip. The field enhancement in this region is maximized in the case of transverse magnetic (TM) modes, for which the major field component is $E_{y}$, perpendicular to the interfaces. For this purpose we focus on three specific aspects of this platform: 1) The confinement factor should be maximized in the region with a minimum effective mode area; 2) The effective index difference between the slab mode of the horizontal slot waveguide without and with a loading material on top has to be maximized to allow small features; 3 ) The structure should be single-mode which is achieved by tuning the loading-strip width and thickness.
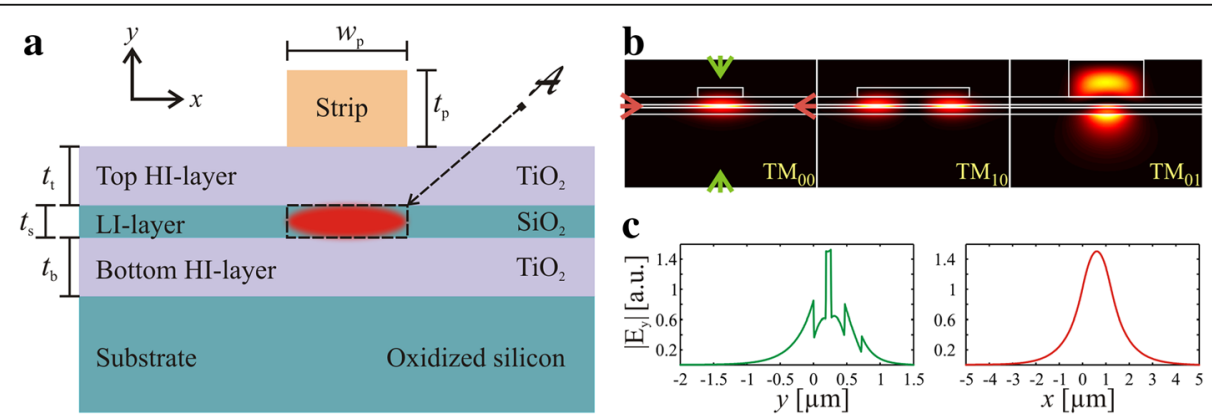

Fig. 1 a Sketch of the strip-loaded slot waveguide. b Power distribution of transverse magnetic modes in the structure. The simulations have been performed for illustration purpose, which means that the strip dimensions have been increased to allow either $\mathrm{TM}_{10}$ or $\mathrm{TM}_{01}$ modes to propagate. $\mathbf{c}$ Mode profiles for $\mathrm{TM}_{00}$ in $y$ - and $x$-directions (indicated by arrows on the $\mathrm{TM}_{00}$ power distribution) 
The analysis of the waveguide structure can be separated into three sub-geometries: the horizontal slot waveguide, the film on top in which the strip will be created, and the loading-strip itself. The first two parts are invariant in the lateral direction, i.e., they are equivalent to a slab waveguide. The third one is acting on the channel waveguide and its optimization, which is our target. Each element influences the behavior of the mode by changing the waveguide characteristics.

\section{Results and discussions Horizontal slot waveguide Simulations}

The horizontal slot waveguide (HSW) is the basis of the structure and it has to be optimized first. The influence of the parameters, i.e., the thickness of the layers, is determined using a mode solver based on the Fourier Modal Method [16-18]. Accurate mode profiles and confinement factors are calculated by Finite Difference mode solver, OptiWave $[19,20]$, in order to avoid ripples due to Gibbs phenomenon inherent to FMM. Our goal is to design a single-mode waveguide (for TM polarization) with the highest relative power confined in the slot.

Titanium dioxide is used as a high-refractive index material $\left(\mathrm{TiO}_{2}, n_{\mathrm{TiO}_{2}}=2.27\right.$ at $\left.\lambda=1550 \mathrm{~nm}\right)$ and silicon dioxide as the low refractive index material for the slot $\left(\mathrm{SiO}_{2}, n_{\mathrm{SiO}_{2}}=1.44\right.$ at $\left.\lambda=1550 \mathrm{~nm}\right)$. The cladding is air and the substrate material is $\mathrm{SiO}_{2}$. These materials are chosen for their refractive index and their transparency in a wide wavelength range, but also for the deposition technique involved in the fabrication (see Methods). The calculations were performed for thicknesses $t_{\mathrm{b}, \mathrm{t}}$ and $t_{\mathrm{s}}$ ranging, respectively, from 100 to $340 \mathrm{~nm}$ for the HIlayers and from 60 to $250 \mathrm{~nm}$ for the slot region. The normalized power $\left(P_{\mathrm{s}}\right)$, also called the confinement factor in the literature, within the slot is chosen as a figure of merit and calculated using Eq. (1) [21].

$$
P_{\mathrm{s}}=\frac{\iint_{\mathrm{A}} P_{z}(x, y) \mathrm{d} x \mathrm{~d} y}{\iint_{\text {total }} P_{z}(x, y) \mathrm{d} x \mathrm{~d} y}
$$

where $P_{z}(x, y)=\Re\left(\mathbf{E}(x, y) \times \mathbf{H}^{\prime \prime}(x, y)\right) \cdot \mathbf{e}_{z}$ is the $z$-component of the Poynting vector, $\mathbf{E}(x, y)$ the electric field and $\mathbf{H}^{\prime \prime}(x, y)$ the complex conjugate of the magnetic field, $\mathbf{e}_{z}$ the unit vector in the $z$-direction, and A symbolizes the region where light has to be confined, i.e., the slot region.

Figure 2a summarizes in a map the normalized power in the slot with the variation of the HI-layers and slot thicknesses. It is evident from this figure that the HI-layers thickness should be set at a value around $200 \mathrm{~nm}$. However, the power is monotonically increasing with the thickness of the slot. This comes from the evident thickness dependence of the confinement factor. To make a relevant use of the map presented in Fig. 2a, one has to consider also the characteristic length discussed in introduction. The hatched region in the figure represents the zone of excluded values, for which the characteristic length is shorter than $t_{\mathrm{s}}$, and therefore the structure is no more a slot waveguide.

Another measure of the efficiency of the structure is the intensity within the slot, which is the power per unit area $I_{\mathrm{s}}=P_{\mathrm{s}} /\left(w_{\mathrm{s}} t_{\mathrm{s}}\right)$. This allows taking into account the geometry of the structure. With this parameter, we see that the trend is reverse when the slot thickness is concerned, a thinner slot enables a larger intensity. Combined with the observation of the variation of power, these results yield a trade-off. One can now set a range of layer thicknesses for which both the power and the intensity are maximized inside the slot region.

The last figures of merit are the effective index and the effective index difference $\left(\Delta n_{\text {eff }}\right)$ for the mode propagating in the slab with and without a cladding material, i.e., the expected effective index contrast of the waveguide. It is important to introduce, already at this stage of the optimization, the loading-strip material, considered, at first, as a semi-infinite medium in the $y$-direction and invariant in the $x z$-plane. In this work the loading-strip
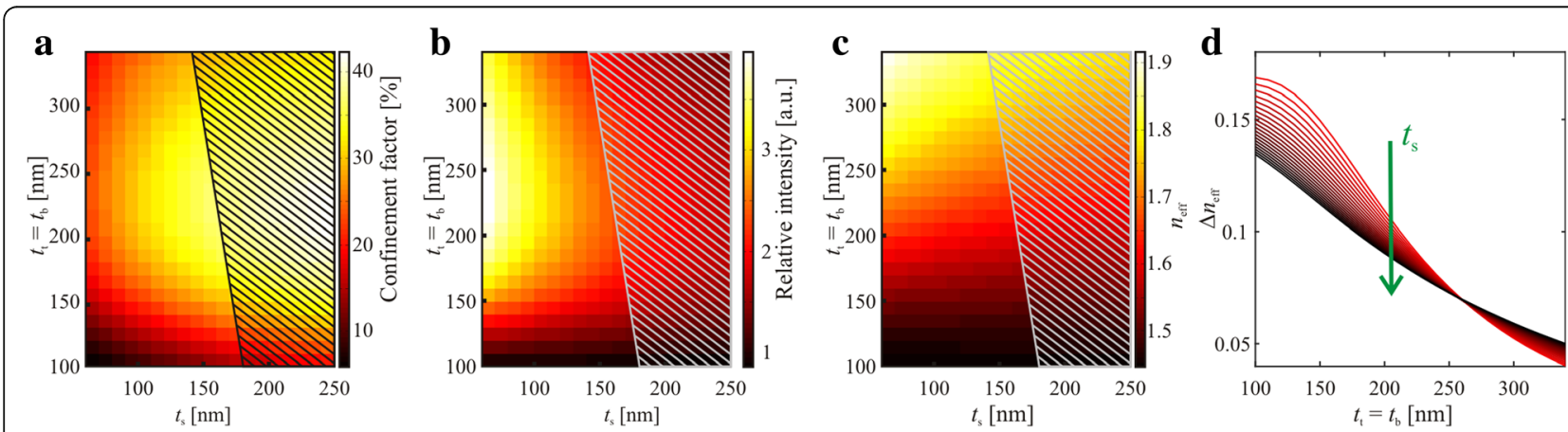

Fig. 2 Optimization of the horizontal slot waveguide: a Confinement factor (normalized power) in the slot region as a function of the thickness of the low index layer (slot) and the HI-layers. $\mathbf{b}$ Intensity in the LI-layer. c Effective index of the fundamental quasi-TM slot mode. The hatched regions exclude thicknesses for which the characteristic length $\left(1 / \gamma_{s}\right)$ is shorter than the slot thickness. $\mathbf{d}$ Effective index difference when the cladding is air and when it is a material with $n_{c}=1.6$ (the green arrow indicates increasing loading strip thickness) 
material is a resist AZ-2070 from MicroChemicals and its refractive index is $n_{c}=1.6$. Results are presented in Figs. $2 \mathrm{c}$ and $\mathrm{d}$, where the thickness of the HI-layers was varied for several slot thicknesses. The largest $\Delta n_{\text {eff }}$ was obtained for the thinnest HI-layers, for which the cladding material will influence more the effective index of the mode. It is evident from these calculations that we need a trade-off between good confinement, high intensity, and large $\Delta n_{\text {eff }}$. Therefore, we chose as the central value, before the finest optimization, an $80 \mathrm{~nm}$-thick LI-layer and $200 \mathrm{~nm}$-thick HI-layers.

An ideal structure would be a slot waveguide suspended in air, leading to a nearly symmetric mode. In a realistic device the waveguide is deposited on a substrate that has to be taken into account in the optimization. In our case, the HI-layers have the strongest influence, among other parameters, on the shape of the slot mode in $y$-direction, i.e., on its symmetry. Depending on the application, one may prefer a slot mode with a profile shifted towards the top HI-layer or towards the bottom one. In this particular study, we are interested in observing the trends in order to show the possibilities offered by the SLSW platform. This is done by varying independently the two thickness of the HI layers around the value determined above. The $\Delta n_{\text {eff }}$ and the confinement factor are used as figures of merit. Results are presented in Fig. 3. Figure 3a shows the influence of the top and bottom HI-layers on the normalized power and Fig. 3b on the effective index difference. It is clear from these results that the bottom layer influences mainly the effective index difference while the top layer has a stronger influence on the field confinement inside the slot. The plots show an almost independence of the two parameters. Note that such a property is directly linked to the range of the parameters. For both HI-layer thicknesses, $\Delta n_{\text {eff }}$ varies linearly and $P_{\mathrm{s}}$ in a parabolic manner. In the region around the central value $(200 \mathrm{~nm}), \Delta n_{\text {eff }}$ increases 4 times faster when $t_{\mathrm{b}}$ decreases and the confinement factor increases 6 times faster when $t_{\mathrm{t}}$ increases. In this work, we target the highest effective index difference while maintaining a reasonable confinement. We determined our targeted parameters for the further fabrication of the horizontal slot waveguide to be: $t_{\mathrm{s}}=80 \mathrm{~nm}, t_{\mathrm{b}}=180 \mathrm{~nm}$ and $t_{\mathrm{t}}=200 \mathrm{~nm}$.

\section{Experimental verifications}

One crucial constraint for the horizontal slot waveguide is the roughness at the interface between different materials. This roughness dictates the propagation losses in the structure. Therefore, we used atomic layer deposition (TFS-200, Beneq) for the fabrication of the different layers constituting the basis of the structure [22-24]. In addition of allowing very smooth and homogeneous layers, this technique provides an accurate control of the thickness over wafer-scale area (see Methods). Layer thicknesses were determined by ellipsometry (VASE, Woollam): $t_{\mathrm{s}}=76 \mathrm{~nm}$, $t_{\mathrm{b}}=185 \mathrm{~nm}$, and $t_{\mathrm{t}}=197 \mathrm{~nm}$. Fig. 4a is an SEM picture of the cross-section of the horizontal slot waveguide.
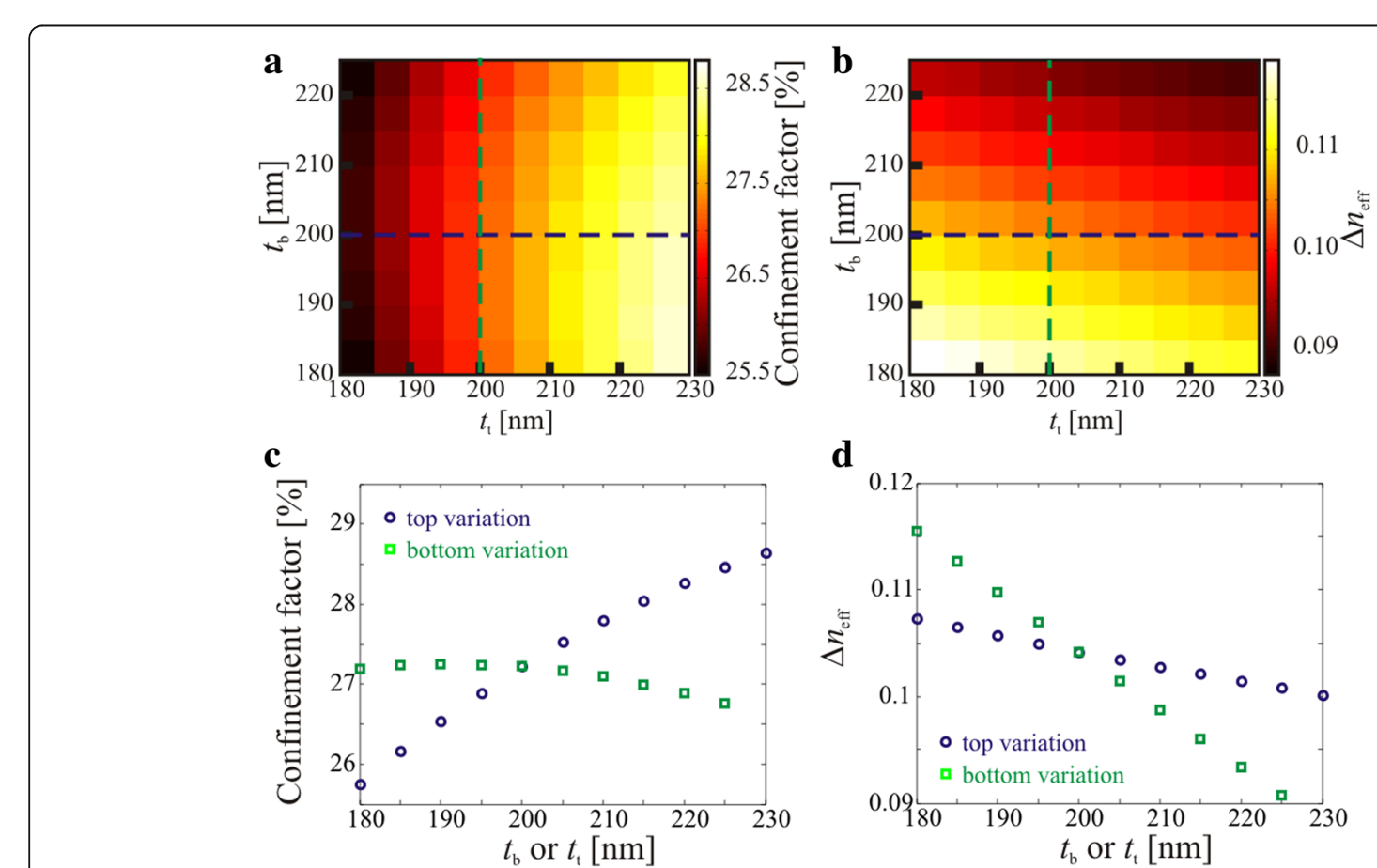

d

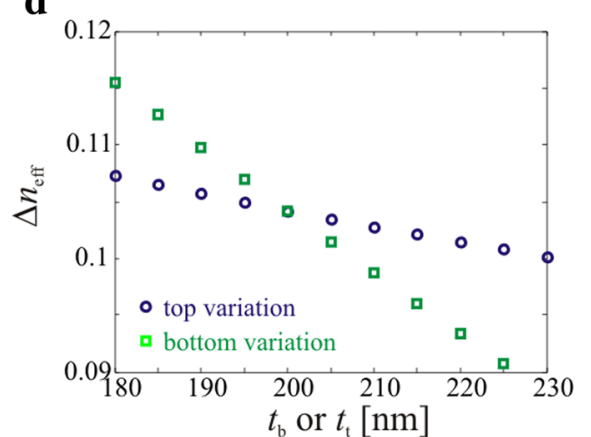

Fig. 3 Influence of the HI-layers thickness on the confinement factor, in \%, of light in the region A (a and $\mathbf{c}$ ) and the effective index difference (b and $\mathbf{d}$ ) of the fundamental slot mode for a stack with and without a loading strip layer 

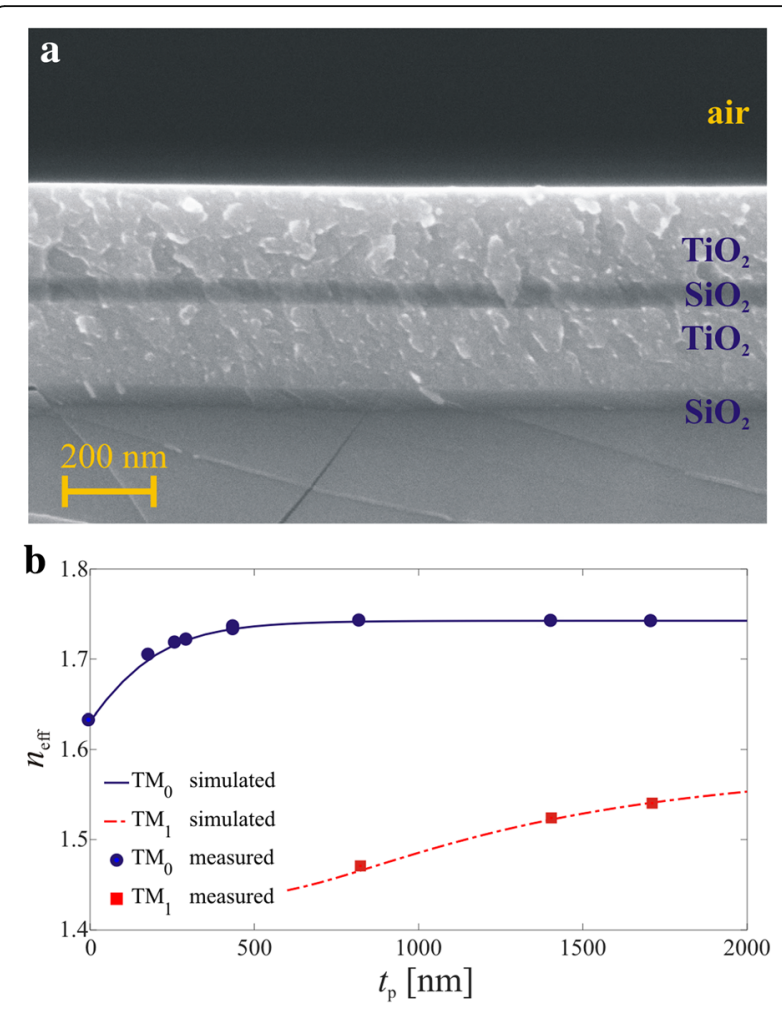

Fig. 4 a SEM micrograph of the ALD layers on an oxidized silicon wafer. Thicknesses measured by ellipsometry are $t_{\mathrm{s}}=76 \mathrm{~nm}, t_{\mathrm{b}}=$ $185 \mathrm{~nm}$, and $t_{\mathrm{t}}=197 \mathrm{~nm}$. The large flake-like structures are due to cleaving. $\mathbf{b}$ Effective indices of the fundamental and the first order TM slab modes of the horizontal slot waveguide for different cladding thicknesses. The curves are the simulated effective indices and the blue dots $\left(T M_{0}\right)$ and the red squares $\left(T M_{1}\right)$ are the measured values

With prism coupler measurements (Metricon M-2010), we confirmed the presence of a single TM mode in the horizontal slot, and determined its effective index $n_{\text {eff }}^{\mathrm{TM}_{0}}=1.63$ at $\lambda=1550 \mathrm{~nm}$. This value is in perfect agreement with our theoretical predictions. To push further our investigations, the influence of the cladding thickness, i.e., the loading-strip layer, was also studied on a test sample coated in the same batch. The sample was coated with an e-beam resist (AZ-2070, from MicroChemicals, $n_{\mathrm{c}}=1.6$ ) by spin-coating and measured with the prism coupler. The resist layer was removed (using the development process involving the developer AR 300-47, AllResist) after each measurement to allow another spin coating with a different thickness. The experimental results, as well as the simulated curves, are presented in Fig. 4b. One can see the first order mode $\left(\mathrm{TM}_{1}\right)$ appearing after a thickness $t_{\mathrm{p}} \approx 550 \mathrm{~nm}$. Note that this mode will correspond further to the $\mathrm{TM}_{01}$ mode in the channel waveguide. The measured effective indices are in good agreement (less than $0.2 \%$ difference) with the calculated ones for both the fundamental and first order TM modes. An important second conclusion about this graph is the invariance of the effective index of the fundamental mode after a certain resist thickness (here, $t_{\mathrm{p}} \approx 480 \mathrm{~nm}$ ). This leads to even more relaxed constraints on the fabrication.

\section{Strip-loaded horizontal slot waveguide Single-mode and multi-mode waveguides}

The loading-strip geometry dictates the properties of the channel waveguide. Its width $\left(w_{\mathrm{p}}\right)$ and thickness $\left(t_{\mathrm{p}}\right)$ are simultaneously studied by FMM. The main figure of merit is the confinement factor of the field in the slot, under the loading-strip (region A). Results are presented in Fig. 5a, where the hatched region is the multi-mode operating zone of the waveguide, which has to be avoided. From the power calculations (Fig. 5a), one can clearly see that the confinement increases with the width of the loading-strip and reaches a maximum for $t_{\mathrm{p}} \approx 250 \mathrm{~nm}$.

A mode extends to infinity, which means that the shape of the guided mode should not modify the losses. However, for integration purpose, the width of the mode is important to 1) determine the position of the neighboring waveguides; 2 ) estimate the width of the sample; 3 ) prevent the bend loss. The shape of the mode profile in $x$-direction depends on the loading-strip width as shown in Fig. 5b. For a narrow loading-strip, the profile tends to be Lorentzian, while it becomes Gaussian-like for wider strips. The full widths at half maximum are: $1.74 \mu \mathrm{m}$ for a $500 \mathrm{~nm}$-wide strip, $1.54 \mu \mathrm{m}$ for $900 \mathrm{~nm}$ wide strip, and $1.54 \mu \mathrm{m}$ for a $1.2 \mu \mathrm{m}$-wide strip. This proves that the loading-strip becomes more efficient in terms of effective index increase when its width increases. These considerations drove us to choose $w_{\mathrm{p}}=1.2 \mu \mathrm{m}$ and $t_{\mathrm{p}}=250 \mathrm{~nm}$ as the loading-strip width and thickness, respectively. For such a waveguide the confinement factor of light in the A region is $20 \%$. It corresponds to an effective mode area of $0.45 \mu \mathrm{m}^{2}$ [25], which is a good value compared to literature considering the low index contrast of the slot waveguide.

Figure 6a is an SEM picture (slightly tilted top view) of the end of the waveguide. In order to show the multimode behavior of the waveguide due to a too wide loading-strip, one observed the modes at the output of a $3 \mu \mathrm{m}$ wide waveguide (Fig. $6 \mathrm{~b}$ ). By tuning the coupling of light in the waveguide, one can excite preferentially the fundamental or the first higher order quasi-TM mode. These observations confirm the above calculations. In Fig. $6 \mathrm{~b}$, the picture of the first higher mode shows a low contrast interference pattern on one side of the mode, where a neighboring waveguide is $200 \mu \mathrm{m}$ away, while on the other side nothing appears (no other waveguide). This pattern is not seen for the 


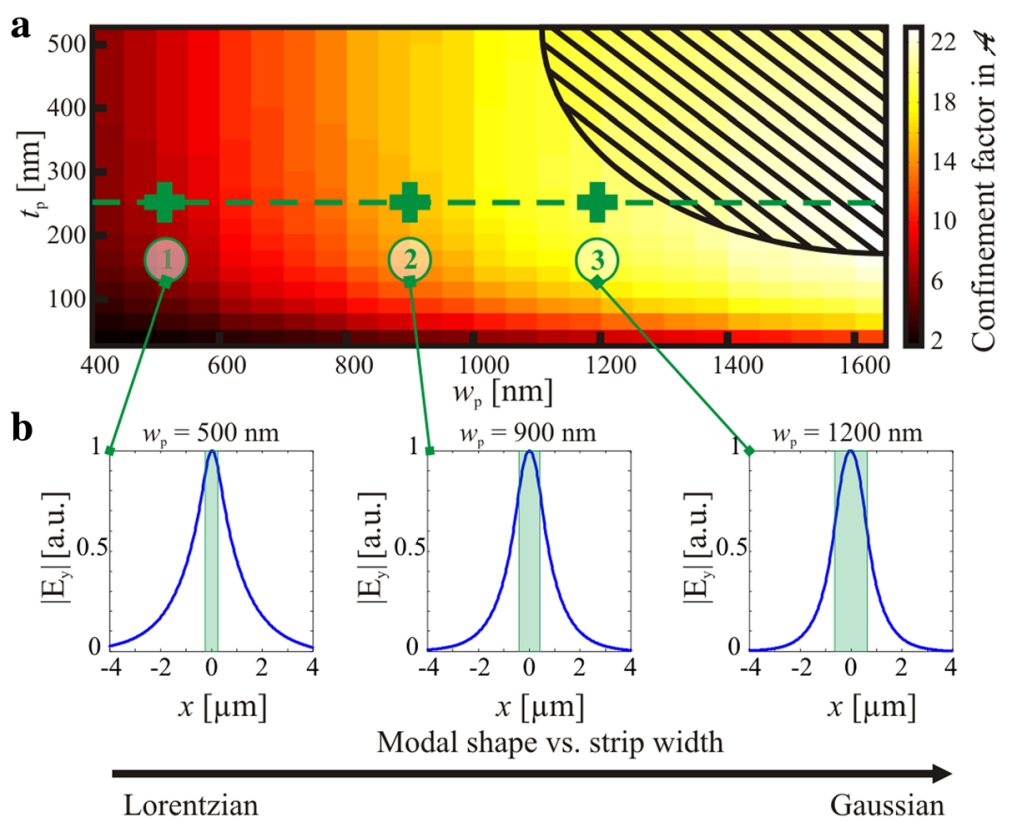

Fig. 5 a Confinement of the fundamental quasi-TM mode inside the SLSW (region A, Fig. 1a) as a function of the width and thickness of the loading-strip. The hatched area shows the multi-mode operating region of the waveguide. $\mathbf{b}$ The $\mathbf{E}_{\mathbf{y}}$ field profiles along the $x$-direction in the center of the slot, corresponding to the green crosses on (a) for different strip widths: $500 \mathrm{~nm}, 900 \mathrm{~nm}$, and $1200 \mathrm{~nm}$ (numbered from 1 to 3) at a constant thickness $t_{\mathrm{p}}=250 \mathrm{~nm}$

fundamental mode proving its confinement and isolation from neighboring waveguides. This emphasizes the role of the shape of the mode, which must be considered during the design.

\section{Propagation loss measurements}

One of the main characteristics of optical waveguides is the propagation loss. Slot waveguides are known to be lossy structures, as already mentioned in the introduction, although significant efforts have been put to solve this problem [26-28].

We determined propagation losses of our single-mode SLSW by the well-known cut-back method [29]. We fabricated first a set of several $4 \mathrm{~cm}$ long waveguides (for statistical purposes) and cleaved them shorter and shorter. For each length the transmitted intensity was measured at $\lambda=1550 \mathrm{~nm}$. A sketch of the optical setup is presented in Fig. 7a.

Although the injection is carefully optimized for each length and each waveguide, some errors may occur due, for instance, to the cleaving of the waveguide. It is thus crucial to measure several waveguides and average the values. More than 20 waveguides and 4 different lengths (from $4.1 \mathrm{~cm}$ to $1.1 \mathrm{~cm}$ ) were investigated. The propagation losses were estimated at $1.4 \pm 0.6 \mathrm{~dB} / \mathrm{cm}$ [Fig. 7b]. This value, already far below standard values for slot waveguides, is still overestimated. Firstly, some stray light cannot be avoided from coupling and shorter the waveguide, stronger its influence on the transmitted intensity; secondly, the coupling is always slightly different and we took it into account with our statistical study.
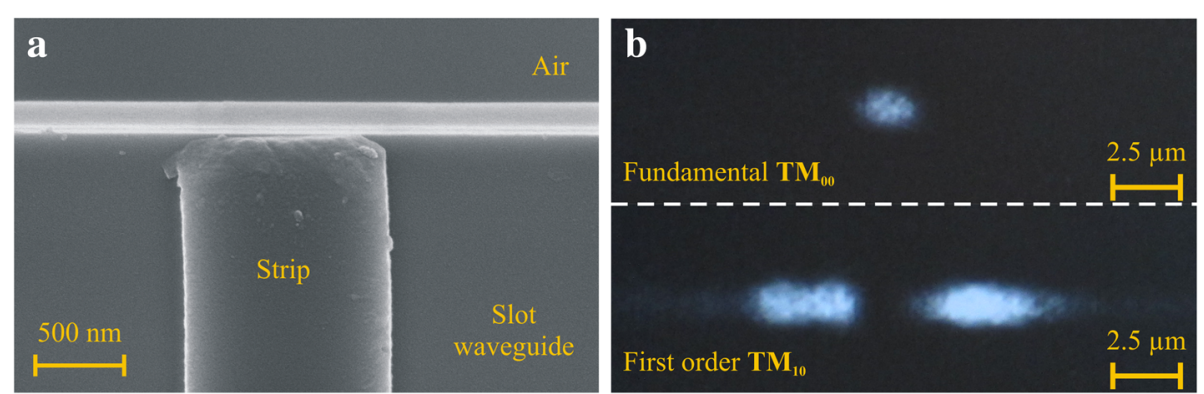

Fig. 6 a SEM micrograph (slightly tilted top view) of a 1.2- $\mu$ m-wide strip patterned on top of the horizontal slot waveguide. b Photographs of the fundamental and the first order transverse magnetic modes at the output of a 3 - $\mu \mathrm{m}$-wide waveguide (objective $\times 60, N . A .=0.85$ ) 

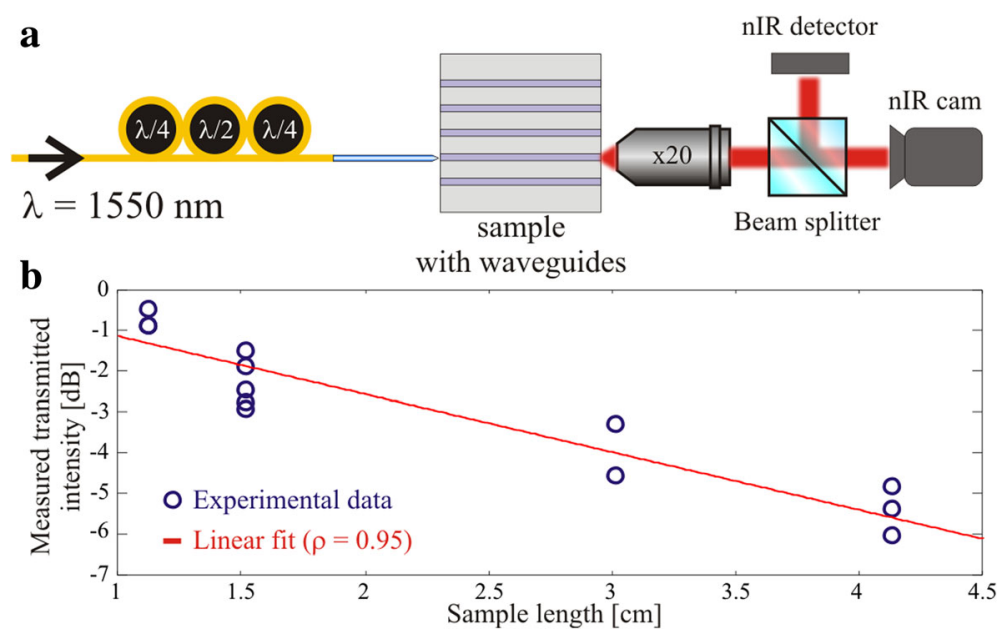

Fig. 7 a Propagation loss setup. b Cut-back method result: transmittance measured at the output of the waveguide as a function of the length of the waveguide (blue circles) and a linear fit (red solid line)

\section{Conclusion}

In this paper, we have presented a thorough investigation of the geometrical parameters of an SLSW. This structure is highly versatile in terms of materials and geometry. We have shown that the high refractive index layers have different influences on the confinement factor and effective index difference, while the loading-strip dictates the number of guided modes. The loading-strip also influences the lateral profile of the mode which is the key for further integration purposes. A precise fabrication, enabled by ALD, determined the low propagation losses $(1.4 \pm 0.6 \mathrm{~dB} / \mathrm{cm})$ compared to the usual values measured for slot waveguides.

\section{Methods}

\section{Numerical methods}

Both Fourier Modal Method (FMM) [16-18] and Finite Difference based mode solver, OptiMode by OptiWave $[19,20]$, are used in this work to determine the optical mode profiles of the waveguides (slab or channels). In both cases a calculation window of $10 \mu \mathrm{m} \times 3.5 \mu \mathrm{m}$ is taken into account with a mesh size of $20 \mathrm{~nm}$ in $x$-direction and $5 \mathrm{~nm}$ in $y$-direction. For FMM calculations, 5 and 200 harmonics are considered to increase the precision of the result and, as mentioned above, reduce the ripples coming from Gibbs phenomenon.

\section{Fabrication techniques}

The slot waveguide layers were coated on a 3-in. oxidized silicon wafer using atomic layer deposition (TFS-200, Beneq). Titanium dioxide layers were deposited at $120{ }^{\circ} \mathrm{C}$, with titanium tetrachloride $\left(\mathrm{TiCl}_{4}\right)$ and water $\left(\mathrm{H}_{2} \mathrm{O}\right)$ as precursors [30]. The silicon dioxide layer was fabricated at $150{ }^{\circ} \mathrm{C}$, with a nitrogen remote plasma to enhance the reactivity of the silicon precursor AP-LTO 330 (Versum Materials) and oxygen $\left(\mathrm{O}_{2}\right)$ as a reactant.

The loading-strip was fabricated directly in the resist AZ-2070 using EBL (Vistec, EBPG 5000+ ES HR).

\section{Characterization setup}

The characterization setup is depicted in Fig. 7a. Polarized light from a tunable laser is coupled to a polarization controller, allowing us to rotate the polarization to the desired one (TM). A polarization maintaining tapered lens fiber with an anti-reflective coating is used to couple light inside the waveguide. The focal spot of the fiber is approximatively $1 \mu \mathrm{m}$ wide, which allows a good overlap with the waveguide mode. The collection is performed using $\mathrm{a} \times 20$ microscope objective. The collimated beam is split in order to measure the output waveguide intensity using a nIR detector (Thorlabs S122C) and, at the same time, constantly monitor the presence of the guided mode.

\section{Abbreviations}

ALD: Atomic layer deposition; EBL: Electron beam lithography; HI: High index; HSW: Horizontal slot waveguide; LI: Low index; SEM: Scanning electron microscope/microscopic; SLSW: Strip-loaded slot waveguide

\section{Availability of data and materials}

The datasets supporting the conclusions of this article are included within the article.

\section{Authors' contributions}

SP performed the simulations, the fabrication, and the characterizations. JL performed the electron beam lithography. MK and SH supervised the work. MR performed some simulations and characterizations, and he initiated the work. SP and MR wrote the manuscript. All the authors read and approved the final manuscript.

\section{Funding}

We acknowledge funding from the Finnish Funding Agency for Innovation under project Tekes FiDiPro NP-NANO (No 40315/13). 


\section{Competing interests}

The authors declare that they have no competing interests.

\section{Publisher's Note}

Springer Nature remains neutral with regard to jurisdictional claims in published maps and institutional affiliations.

Received: 6 October 2017 Accepted: 13 November 2017

Published online: 21 November 2017

\section{References}

1. Almeida, V.R., Xu, Q., Barrios, C.A., Lipson, M.: Guiding and confining light in void nanostructure. Opt. Lett. 29, 1209-1211 (2004)

2. Barrios, C.A., Sánchez, B., Gylfason, K.B., Griol, A., Sohlström, H., Holgado, M., Casquel, R.: Demonstration of slot-waveguide structures on silicon nitride / silicon oxide platform. Opt. Express. 15, 6846-6856 (2007)

3. Koos, C., Vorreau, P., Vallaitis, T., Dumon, P., Bogaerts, W., Baets, R., Esembeson, B., Biaggio, I., Michinobu, T., Diederich, F., Freude, W., Leuthold, J.: All-optical high-speed signal processing with silicon-organic hybrid slot waveguides. Nat. Phot. 3, 216-219 (2009)

4. Robinson, J.T., Chen, L., Lipson, M.: On-chip gas detection in silicon optical microcavities. Opt. Express. 16, 4296-4301 (2008)

5. Barrios, C.A., Gylfason, K.B., Sánchez, B., Griol, A., Sohlström, H., Holgado, M., Casquel, R.: Slot-waveguide biochemical sensor. Opt. Lett. 32, 3080-3082 (2007)

6. Dell'Olio, F., Passaro, V.M.N.: Optical sensing by optimized silicon slot waveguides. Opt. Express. 15, 4977-4993 (2007)

7. Salih, M., Janani, K., Chen, X., Jacobson, E., Gautam, S., Mickelson, A.: Losses of slot mode devices. J. Lightwave Technol. 34, 3901-3907 (2016)

8. Baehr-Jones, T., Hochberg, M., Walker, C., Scherer, A.: High-Q optical resonators in silicon-on-insulator-based slot waveguides. Appl. Phys. Lett. 86, 081101 (2005)

9. Xiong, C., Pernice, W.H., Li, M., Tang, H.X.: High performance nanophotonic circuits based on partially buried horizontal slot waveguides. Opt. Express. 18, 20690-20698 (2010)

10. Xu, Q., Almeida, V.R., Panepucci, R.R., Lipson, M.: Experimental demonstration of guiding and confining light in nanometer-size low-refractive-index material. Opt. Lett. 29, 1626-1628 (2004)

11. Sun, R., Dong, P., Feng, N.-n., Hong, C.-y., Michel, J., Lipson, M., Kimerling, L.: Horizontal single and multiple slot waveguides: optical transmission at $\lambda=$ $1550 \mathrm{~nm}$. Opt. Express. 15, 17967-17972 (2007)

12. Preston, K., Lipson, M.: Slot waveguides with polycrystalline silicon for electrical injection. Opt. Express. 17, 1527-1534 (2009)

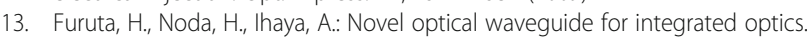
Appl. Opt. 13, 322-326 (1974)

14. Roussey, M., Ahmadi, L., Pélisset, S., Häyrinen, M., Bera, A., Kontturi, V., Laukkanen, J., Vartiainen, I., Honkanen, S., Kuittinen, M.: Strip-loaded horizontal slot waveguide. Opt. Lett. 42, 211-214 (2017)

15. Lipson, M.: Guiding, modulating, and emitting light on silicon - challenges and opportunities. J. Lightwave Technol. 23, 4222-4238 (2005)

16. Li, L.: Use of Fourier series in the analysis of discontinuous periodic structures. J. Opt. Soc. Am. A. 13, 1870-1876 (1996)

17. Tervo, J., Kuittinen, M., Vahimaa, P., Turunen, J., Aalto, T., Heimala, P., Leppihalme, M.: Efficient Bragg waveguide grating analysis by quasi-rigorous approach based on Redheffer's star product. Opt. Comm. 198, 265-272 (2001)

18. Hugonin, J., Lalanne, P., Del Villar, I., Matias, I.: Fourier modal methods for modeling optical dielectric waveguides. Opt. Quant. Electron. 37, 107-119 (2005)

19. Lüsse, P., Stuwe, P., Schüle, J., Unger, H.-G.: Analysis of vectorial mode fields in optical waveguides by a new finite difference method. J. Lightwave Technol. 12, 487-493 (1994)

20. https://optiwave.com/ (06/28/2017)

21. Robinson, J.T., Preston, K., Painter, O., Lipson, M.: First-principle derivation of gain in high-index-contrast waveguides. Opt. Express. 16 16659-16669 (2008)

22. Leskelä, M., Ritala, M.: Atomic layer deposition (ALD): from precursors to thin film structures. Thin Solid Films. 409, 138-146 (2002)

23. Ritala, M., Niinistö, J.: Atomic Layer Deposition in Jones, a.C., and Hitchman, M.L.: Chemical Vapour Deposition: Precursors, Processes and Applications, pp. 158-206. The Royal Society of Chemistry, Cambridge (2009)
24. Miikkulainen, V., Leskelä, M., Ritala, M., Puurunen, R.L.: Crystallinity of inorganic films grown by atomic layer deposition: overview and general trends. J. App. Phys. 113, 021301 (2013)

25. Rukhlenko, I.D., Premaratne, M., Agrawal, G.P.: Effective mode area and its optimization in silicon-nanocrystal waveguides. Opt. Lett. 37, 2295-2297 (2012)

26. Spott, A., Baehr-Jones, T., Ding, R., Liu, Y., Bojko, R., O'Malley, T., Pomerene, A., Hill, C., Reinhardt, W., Hochberg, M.: Photolithographically fabricated low-loss asymmetric silicon slot waveguides. Opt. Express. 19, 10950-10958 (2011)

27. Alasaarela, T., Korn, D., Alloatti, L., Säynätjoki, A., Tervonen, A., Palmer, R., Leuthold, J., Freude, W., Honkanen, S.: Reduced propagation loss in silicon strip and slot waveguides coated by atomic layer deposition. Opt. Express. 19, 11529-11538 (2011)

28. Häyrinen, M., Roussey, M., Säynätjoki, A., Kuittinen, M., Honkanen, S.: Titanium dioxide slot waveguides for visible. Appl. Opt. 54, 2653-2657 (2015)

29. Vlasov, Y., McNab, S.: Losses in single-mode silicon-on-insulator strip waveguides and bends. Opt. Express. 12, 1622-1631 (2004)

30. Häyrinen, M., Roussey, M., Bera, A., Kuittinen, M., Honkanen, S.: Atomic Layer Re-Deposition for Nanoscale Devices in Leon Shohet, J.: Encyclopedia of Plasma Technology, pp. 143-151. Taylor and Francis, Abingdon (2017)

\section{Submit your manuscript to a SpringerOpen ${ }^{\circ}$ journal and benefit from:}

- Convenient online submission

- Rigorous peer review

- Open access: articles freely available online

- High visibility within the field

Retaining the copyright to your article

Submit your next manuscript at $>$ springeropen.com 